\title{
The kinesthetic fusion effect: Perceptual elimination of spatial discordance in the kinesthetic modality
}

\author{
F. T. KENNY and B. CRASKE \\ Faculty of Medicine and Department of Psychology, Memorial University of Newfoundland \\ St. John's, Newfoundland AIB 3X9, Canada
}

\begin{abstract}
Three experiments in intrasensory kinesthetic discordance are reported. The treatment required both arms to be outstretched in the midsagittal plane. In the absence of vision, a finger of one hand depressed a button that caused a probe to touch the other arm $12.7 \mathrm{~cm}$ closer to the body than the position of the finger. This difference was at first clearly sensed, but very rapidly the disparate positions were perceived to be identical: We have called this "the kinesthetic fusion effect." It was also shown that a delay of up to 4 sec in probe onset after the finger depressed the button did not diminish the extent of fusion. The phenomenon gave rise to a predictable aftereffect, in which a probe directly opposite to the active finger was sensed to be farther away than the finger. The results were not due either to joint adaptation or to postural aftereffects.
\end{abstract}

Consideration of the properties of human localization systems shows quite clearly that they are both plastic and capable of functional adaption and modification. Such functions as visual and auditory direction and registered limb position have adjustable parameters (Welch, 1978), with adjustment occurring in a number of ways. For example, there is ample evidence that previous posture of the eye or limb can, for a short time, bias the accuracy of registration of subsequent postures (Nachmias, 1953; Park, 1969). It is also known that asymmetrical visual input can lead to changes in egocentric localization of objects in the field of view (Bruell \& Albee, 1955) and that the simultaneous presence of two separated visual and auditory stimuli can affect the perceived location of each (Radeau \& Bertleson, 1974).

These examples indicate the ready plasticity of our spatial senses, yet they would not be appropriately classified as adaptive, for there is little behavioral advantage to be gained from their occurrence. Nevertheless, there is a wealth of situations requiring functional adaptation. For example, consider the effect of bodily growth on the spatial relationships among eyes, ears, and joints. These are constantly changing, inevitably leading to intersensory discordance. Yet, the organism still needs to locate objects accurately in space. That is to say, objects must occupy only one position, irrespective of how many sensory channels are in use. This can be achieved if all systems that register spatial information cross-check one another and adjust appropriately to maintain intermodal unity.

The rapid elimination of sensory discordance (adaptation) is thus the mechanism by which the fun-

This research was supported in part by MRC Grant MA-6696. damental and underlying plasticity of the spatial senses leads, not to chaos, but to the day-to-day working integration and stability of the system. Any drift away from the veridical that may occur in a given sense will be reset automatically by the resultant increase in discordance. Hence, the overall system property that is argued to cope with the sensory problems associated with growth predicts a powerful adaptive effect as a result of sensory discordance, irrespective of its genesis. Much of the work on adaptation to prismatic displacement can be understood as discordance-driven adaptation. Thus, prismatically induced visual kinesthetic discordance is a sufficient condition for oculomotor adaptation of visual direction (Craske \& Crawshaw, 1978).

Literature on the topic of spatial localization always refers to visual and auditory direction and articular position sense. One crucial element that is largely ignored, however, is that of registered limb length. It is clear that information about limb length is required for accurate reaching to occur in the absence of visual guidance, and it is also clear that the stored length or current information about the length of a limb or a segment must be capable of adjustment to compensate for growth. We would therefore expect registered limb length to be intrinsically plastic and capable of adaptation as a result of imposed discordance.

The use of the term "kinesthesis" in this paper should be made clear. An accepted definition, from Howard and Templeton (1966), is "the discrimination of the positions and movements of body parts based on information other than visual, auditory, or verbal." Here we are interested in the positional aspects. Nonvisual knowledge of the position of a particular point on a limb (e.g., a fingertip) requires 
the brain to know each of the associated joint angles and the lengths of each of the articulating segments. That is, kinesthesis necessarily involves analysis of length. The presence of a stimulus on the skin can yield positional information. In order for this to occur, information from the tactile sheet must be mapped onto the length domain, and in this way stimuli transduced by the tactile sheet fulfill a kinesthetic function.

The first hint that there could be adaptive change in registered arm length came from an extensive series of experiments carried out in our laboratories. These asked whether, when movement was restricted to the elbow, prism adaptation of perceived elbowjoint angle would occur. The experiments failed to demonstrate any such prism aftereffects but did suggest the occurrence of a change in perceived arm length (Kenny, 1978). This, presumably, was an attempted adaptive response in the absence of change in perceived joint angle.

This observation led to studies that deliberately introduced intramodal discordance of perceived length between the two arms. This was achieved by a device with a push button on one side and probes that could emerge from the other side. The subject held the left arm extended along the probe side and pressed the button with a finger of the right (extended) hand and arm. A probe could stimulate the left arm at a point that was not opposite to the button but was closer to the trunk, thereby creating fingertip-to-probed-spot discordance. The probe opposite to the fingertip could also be separately activated.

\section{Kinesthetic Fusion Effect}

Pilot trials with this device (using the authors as subjects) revealed a curious phenomenon. When the blindfolded subject swung his arm over a short distance and depressed the button, he was distinctly aware of an initial discrepancy between the point at which his finger hit the button and the point at which the probe struck his other arm. With trials every 2-3 sec, the pressing finger and probed position began to feel closer together, until (usually within 10 trials, but sometimes after up to 15 or 20) a stage was reached in which the finger and probed spot felt directly opposite to each other. This perceptual change, termed the "kinesthetic fusion effect," persisted, provided that the subject continued to press the button regularly, and it occurred even when a subject was (visually) familiar with the apparatus and its mode of operation. However, when a subject was permitted to see his arm and hands while operating the device, fusion did not occur. ${ }^{1}$

The kinesthetic fusion effect appeared to be a perceptual concomitant of adaptation to the imposed spatial discordance and should therefore produce an aftereffect. After fusion takes place, if the subject, when next pressing, stimulates a point on his other hand directly opposite to the button, then the perceptual experience should be that the probed spot feels farther away than does the pressing finger. If a subject continues to press the button with the probe directly opposite to it, eventually a reversal of the effects of the initial spatial discordance should occur, so that the probe and finger gradually and correctly come to feel opposite to each other.

\section{EXPERIMENT 1}

\section{Method}

Subjects. Twenty-four paid volunteer subjects (university students) of both sexes participated in the experiment. Subjects served as their own controls, each receiving one experimental and two control sessions at least $24 \mathrm{~h}$ apart according to a predetermined random order. All subjects were given instructions on operating the equipment and making spatial estimates (see below). However, no potential perceptual changes were suggested.

Apparatus. Two solenoid-activated probes were positioned at arms' length on the right-hand side of a Plexiglas plate that was in the subject's median sagittal plane (Figure 1). They were electrically operated by means of a microswitch behind a push button. One probe (P1) was mounted directly opposite to the center of the microswitch button, while the second probe (P2) was mounted $5 \mathrm{in} .(12.7 \mathrm{~cm})$ closer to the subject's body. Only one probe could be activated by the subject at any given time. Prior to the first session, the subject was shown photographs of the apparatus (without the probes) as a guide to where he was to position the arms and how he was to move the right arm.

Procedure. The subject was blindfolded and stood holding the left arm extended straight in front of him with the hand in the median sagittal plane. The middle finger rested in a slot in a backstop, and the left hand, wrist, and forearm were flush along the left side of the Plexiglas plate. The right arm was also held extended straight out, but initially was adducted to a rest position approximately $30 \mathrm{deg}(.52 \mathrm{rad})$ to the right of the center line.

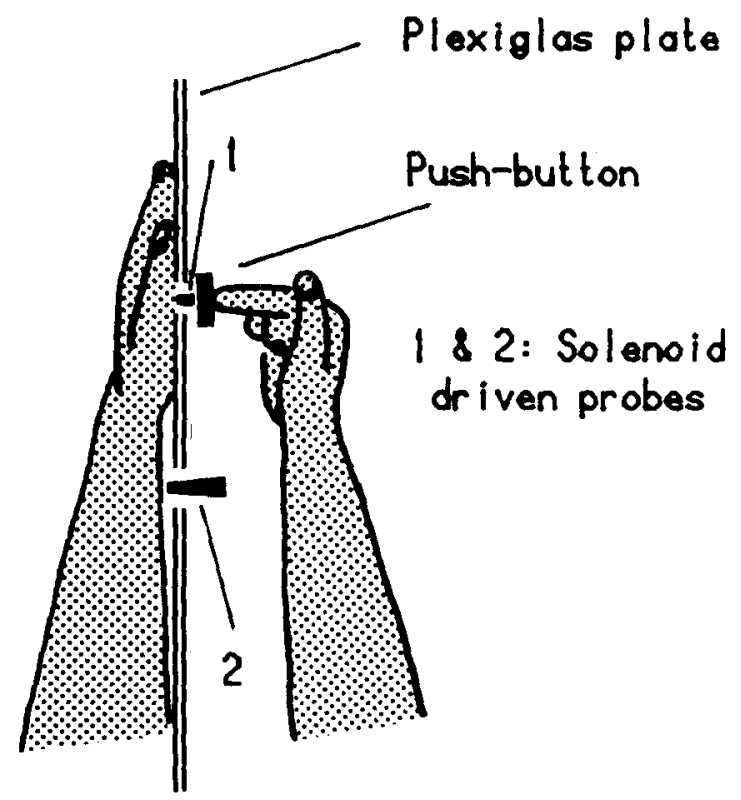

Figure 1. Apparatus used to produce kinesthetic fusion. The experimenter could select which of the two probes was to be actuated. 
The right hand was held in a fist, with the exception of the index finger, which was partially flexed so that it pointed toward the button. The experimenter ensured that the subject's shoulders were held straight along the coronal plane.

On each trial, the subject swung the right arm toward the button and depressed it, thereby activating one of the probes, which protruded through a slot in the Plexiglas panel and struck the left hand or wrist. The subject paused momentarily when he had depressed the button and gave a verbal estimate (in inches) of the perceived distance between the pressing finger and the point at which the probe was hitting the left arm (called the probe/ finger distance). The subject said "behind," "opposite," or "ahead," to indicate whether the probe position felt closer to, the same distance from, or farther from the body than the pressing finger position.

Once a subject had made his verbal estimate, he released the button, swung the right hand back to the rest position, and immediately initiated a new trial. The subject paced himself, but the total time taken for a trial was usually 2 to $3 \mathrm{sec}$. In each session, the subject received 10 blocks of 30 trials each, with a $60-\mathrm{sec}$ rest interval between each block. In the experimental condition, the second probe (P2) was used for the first 20 trials of each block, which were discordance trials, and P1 (the opposite probe) was used for the last 10 trials. In the first control condition $(\mathrm{Cl}$, no discordance), the opposite probe was used for the entire 30 trials, while in the second control condition ( $\mathrm{C} 2$, all discordant), the nearer probe was used for the entire 30 trials. In the experimental condition, it was predicted that, on the 21 st trial, the opposite probe would feel farther away than the pressing finger, this aftereffect decaying during the $\mathbf{1 0}$ posttreatment trials. No perceived changes were predicted during $\mathrm{Cl}$. The second control condition (C2) was employed to assess any cumulative effect due to continual discordance. Specifically, it was predicted that there would be a decrease in the perceived probe/finger distance across trials in all blocks and that this would be smaller during the 10th block of trials as compared with the first.

\section{Results}

Figure 2 shows the changes in mean estimates of the probe/finger distance for the last 10 trials in the experimental and first control $(\mathrm{Cl})$ condition, when the probe and finger were truly opposite to each other. When the probe was perceived "ahead" of the finger, judgments were given positive values; "behind" the finger judgments were given negative values. It can be seen that an overestimate of probe

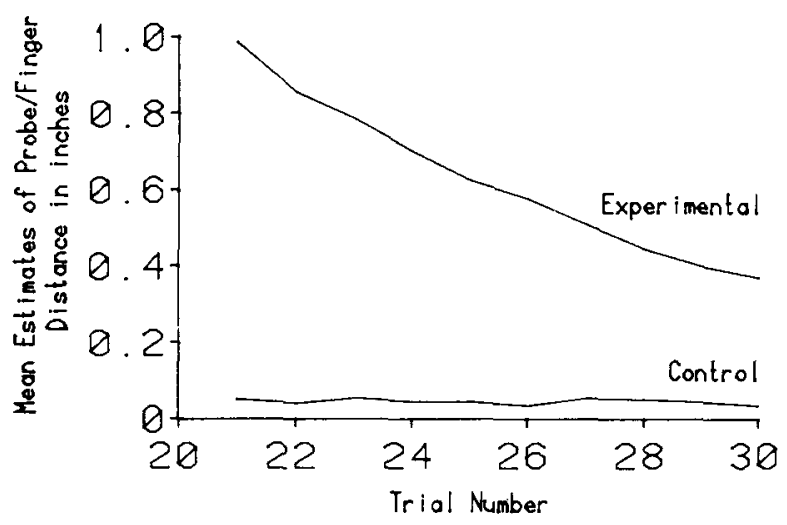

Figure 2. Change over 10 trials in mean $(N=24)$ estimate of probe/finger distance when probe and finger are opposite to each other following 20 discordant (experimental) or 20 concordant (control) trials.

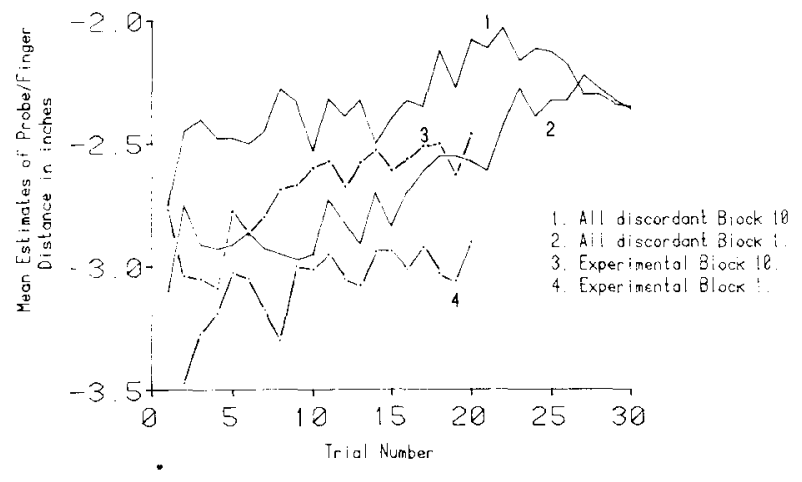

Figure 3. Change over treatment trials in mean estimate of probe/finger distance when probe and finger are separated by 5 in. Blocks 1 and 10 are shown for each of two conditions (all discordant: $N=11,1$ outlier rejected; experimental: $N=12$ ). Each group consists of those subjects who carried out the relevant condition first or after the $\mathrm{C} 1$ (zero-discordance) condition.

position with respect to the finger took place. After 20 trials with an actual probe/finger distance of -5 in. $(-12.7 \mathrm{~cm})$, the estimated probe/finger distance on the 21 st trial in the experimental condition was 1 in. $(2.54 \mathrm{~cm})$. During the 10 posttreatment trials (21st to 30 th trials), in which probe/finger distance was zero, the aftereffect decreased but did not disappear.

A two-way analysis of variance yielded significant main effects for conditions (experimental/C1) $[F(1,23)=29.12, p<.001]$ and repeated measures (trials) $[F(9,120)=18.36, p<.001]$, as well as a significant interaction (Condition by Trials) $[F(9,120)=$ 23.24, $\mathrm{p}<.001]$. A test of simple main effects across the last 10 trials in the experimental condition gave $F(9,120)=23.30, p<.001$, showing that a significant reduction in the estimated probe/finger distance did, in fact, take place. Further analyses were considered unnecessary at this stage.

In the second control condition ( $\mathrm{C} 2$ ), all trials were discordant, so substantial fusion effects would be predicted. Twelve of 24 subjects reported complete kinesthetic fusion at some point during each block of 30 trials with P2. This percentage roughly accords with that reported in previous experiments (Kenny, 1978). The remaining 12 subjects reported varying degrees of partial fusion. Figure 3 illustrates that, in both the experimental and the all-discordant conditions, there was a regular decrease in perceived probe/ finger distance within a block of trials and a cumulative effect across blocks.

In essence, then, during no-discordance trials $(\mathrm{Cl})$, perceptual modification does not occur. Discordance trials (experimental) lead to experienced fusion and subsequent overestimates of the location of the opposite probe with respect to the finger; these decay with repeated pressing over time. During alldiscordance trials (C2), the tendency to fusion increases with trials and over blocks. 


\section{EXPERIMENT 2}

There is always the possibility that experiments involving reaching and spatial discordance may lead to postural aftereffects or adaptive effects associated with changes in registered joint angle. Should they occur during kinesthetic fusion, such effects might sum with any other changes or even constitute the major effect. For example, if, in the fusion situation, we consider the feet remaining still and the body in the anatomical position, we can envisage that the experience of fusion may be the result of a perceived (but nonveridical) rotation of the hips with respect to a line drawn between the feet. In addition, an independent apparent rotation of the whole shoulder girdle with respect to the trunk is also possible, as are changes in registered shoulder-joint angle for each of the arms. Thus, the second experiment looked for the occurrence of postural or joint aftereffects. ${ }^{2}$ It also investigated whether kinesthetic fusion requires a temporal coincidence of the termination of movement of one arm with the probing of the other.

\section{Method}

Subjects. There were 37 paid volunteer subjects.

Apparatus. The experimental apparatus was essentially the same as in Experiment 1 except that a single solenoid-activated probe was mounted 4 in. $(10.2 \mathrm{~cm})$ closer to the subject's body than was the button. Each subject stood on an adjustable platform in front of the apparatus. Mounted immediately above the platform was an electrically wound camera that was appropriately aligned and focused to photograph the bare shoulders and outstretched arms of the subject, who wore a sleeveless athletic shirt.

Prior to each experimental session, a large cross was marked with a felt-tip pen near the acromio-clavicular joint of each of the shoulders and near the biceps tendon in the crook of each elbow. The subject was raised so that his outstretched arms could rest on a foam-covered platform (which held the discordance apparatus) at approximately $21 \mathrm{~cm}$ below shoulder height in the median sagittal plane. His feet were then positioned against a toe board (legs slightly apart) and were clamped to keep them in a fixed position.

The discordance apparatus was positioned along the median plane and could be adjusted with respect to the two outstretched arms so that, as both arms completed adducting movements, ${ }^{3}$ the flexed index finger of the right arm would touch the button, and the fingertips of the left arm would brush against a backstop and come to rest against the Plexiglas plate. With this procedure, both arms would come to rest symmetrically about the median sagittal plane, with the shoulders parallel to the front of the platform, and the probe would strike approximately the midpoint of the left wrist. The extent of subsequent horizontal adduction of each arm was limited by foam armstops. The total excursion for each arm was thus about $30 \mathrm{deg}$ (.52 rad).

Procedure. Before the discordance trials commenced and after every subsequent block of trials, two photographs were taken: (1) subject standing normally and comfortably with arms at sides; and (2) subject holding arms "straight out." The order of photographs was alternated at the end of each block and was counterbalanced across subjects.

Each subject received a single experimental session consisting of seven blocks of trials. In each trial, beginning with both arms resting on the platform and against the stops, subjects adducted their outstretched arms about the shoulder joint, with the index finger of the right hand subsequently touching the button and the probe being pushed into the left wrist. Subjects then gave a verbal estimate of the perceived distance between finger and probe before returning their arms to the starting position. Subsequent trials were performed on a self-paced basis approximately every $2-3 \mathrm{sec}$ until a total of 15 had been completed. Rest intervals of $60 \mathrm{sec}$ were given between blocks of trials.

In the first two blocks of trials, there was no delay between the buttonpress and subsequent probing. In blocks 3 and 4 , there was a 2-sec delay, and in blocks 5 and 6 , there was a 4-sec delay. In the seventh (and final) block, there was again no delay. This "shaping procedure" for the first six blocks was adopted in order to create optimal conditions for fusion, since nothing was known about the likelihood of fusion with delay. A return to a no-delay condition in the seventh block was made, since Experiiment 1 , which showed a practice effect across blocks of trials, implied that fusion would be strongest at this stage. During the delay trials, the subject pressed the button and kept his finger in place until the probe hit his left arm.

\section{Results}

All but two of the 37 subjects showed substantial fusional changes across the seven blocks of trials. However, since there was not full fusion in all instances, it was decided to adopt a "strong" criterion when analyzing the results for postural/joint aftereffects. This was defined as a subject's achieving full fusion or giving judgments within $1 / 2$ in. $(1.27 \mathrm{~cm})$ of this in at least one of the conditions. Of the 17 subjects who met this criterion, 11 attained it in the first no-delay condition, 9 in the 2-sec delay condition, 16 in the 4-sec delay condition, and 14 in the final no-delay condition.

The photographs of these 17 subjects were examined, and the angular deviation between observed shoulder axis and a reference line parallel to the front of the apparatus was measured to within $.5 \mathrm{deg}(.0087$ rad). The deviations of the lines joining shoulder to elbow were similarly measured with respect to a reference line perpendicular to the shoulder axis line. The means of these deviations for each condition are shown in Table 1.

There were no significant differences between pretreatment and posttreatment values for shoulder orientation and left-arm position $[\mathrm{F}(4,64)=.09$ and 1.04, respectively]. A significant effect was found for the right arm $[F(4,64)=3.34, p<.05]$, and subsequent tests showed that this represented a small but

Table 1

Relationship to Reference Line in Pretest and After Three Levels of Delay

\begin{tabular}{lccc}
\hline & \multicolumn{3}{c}{ Mean Deviation (in Degrees) } \\
\cline { 2 - 4 } Condition & Shoulders & Right Arm & Left Arm \\
\hline Pretest & -.12 & 89.0 & 92.1 \\
0-Sec Delay & .19 & 87.0 & 91.4 \\
2-Sec Delay & -.18 & 87.7 & 91.1 \\
4-Sec Delay & .09 & 88.6 & 90.9 \\
\hline
\end{tabular}

Note-Angles were measured clockwise from the reference line. 
significant 2 -deg $(.035 \mathrm{rad})$ clockwise shift between pretreatment measures and the first no-delay condition $[t(16)=3.08, p<.01]$. No other shifts were significant.

The behavioral significance of this right-arm shift is questionable, since it does not exhibit itself again, even after the 7th block of trials (no delay) in which the strongest fusional responses occurred. In general, it is clear that postural aftereffects are not present and are unlikely as a source of explanation for kinesthetic fusion. Furthermore, it is difficult to see how such a shift in perceived relationship between arm and shoulder could, on its own, be directly related to the perceived fusion effect in which the finger feels directly opposite to the left wrist.

It is also clear that a delay between buttonpressing and subsequent probing does not preclude a fusional shift. Indeed, 8 of 9 subjects who met the strong criterion in the 2 -sec delay condition showed complete fusion, and 12 of 16 subjects showed full fusion in the 4-sec delay condition.

It was decided to compare the extent to which fusion could occur under delay and no-delay conditions. The only way in which two means could be established such that the number of preceding trials remained constant was as follows. Using data from all 37 subjects, two means were derived by combining the means for the fusional shift on the last trial ${ }^{4}$ of the first and seventh blocks (no-delay conditions) and comparing this with the mean shift in the last trial of the third and fifth blocks (delay conditions). Thus, 118 trials were associated with each mean.

Mean shifts of $1.9 \mathrm{in} .(4.8 \mathrm{~cm})$ for the no-delay conditions and 1.8 in. $(4.57 \mathrm{~cm})$ for the delay conditions were not significantly different $[t(36)=1.9$, $\mathrm{p}>.05$, illustrating that delay does not preclude a fusional shift, and it does not affect the magnitude of the shift.

\section{EXPERIMENT 3}

In order to establish that the fusion effect cannot be explained by postural adaptation, one further experiment was required. This examined the possibility of a perceived "superflexion" of the index finger of the pointing hand accounting for the experience of fusion and the reported aftereffect. It is logically possible that the subject gradually experiences adaptation of position sense at the joints of the phalanges of the index finger and between the first phalanx and metacarpus. Under these circumstances, instead of correctly feeling the finger to be turned through $90 \mathrm{deg}$, a much larger angular excursion would be perceived. This form of adaptation could lead to the right fingertip being sensed as close to or opposite to the probe. This would account for fusion and subsequent aftereffect. An experiment was carried out in which no flexion of the fingers was permitted during either treatment or testing.

\section{Method}

Subjects. Twenty-two subjects of both sexes participated in this experiment.

Apparatus. This was essentially the same as in Experiment 1, except that the button was arranged so that a finger would touch it when the hand was semipronated (plane of hand was vertical) and the fingers were extended. The left arm could be probed either 5 in. $(12.7 \mathrm{~cm})$ closer to the body than the right fingertip or opposite to it.

Procedure. In the experimental condition, 11 subjects pressed the button with the right hand (which was oriented as before, except that the fingers were extended) for 20 trials and were probed on the left arm at a position 5 in. $(12.7 \mathrm{~cm})$ closer to the body. On the 21st press, they received the "opposite" probe. In the control condition (also 11 subjects), the first 20 presses resulted in activation of the "opposite" probe, as did the 21 st press. Six blocks of trials were given in each case, and spatial estimates were required as in Experiment 1.

\section{Results and Discussion}

The experimental group showed a perceived intramodal aftereffect of $1.16 \mathrm{in}$. $(2.95 \mathrm{~cm})$ in the predicted direction and reported no perceived flexion of the phalanges. The control group showed almost no error: mean $=-.06$ in. $(-.15 \mathrm{~cm})$. After square-root transformation to equate variances, the two means are shown to be significantly different $[F(1,120)=$ $9.49, \mathrm{p}<.01]$. A subsequent test of strength of association showed that $28 \%$ of the variance was accounted for by the experimental manipulation.

The data from the present experiment (fingers not flexed) are not strictly comparable to those of the first (fingers flexed); however, the magnitude of the aftereffect is very similar. It is clear from these results that flexion or extension of the fingers does not affect the occurrence of the fusion phenomenon.

\section{GENERAL DISCUSSION}

These three experiments show that when there is an intramodal discordance of kinesthetic information about length (or extent), the brain acts to eliminate the sensed discrepancy, resulting in perceived fusion or identity for disparate positions along each arm. Furthermore, this leads to a predictable aftereffect: When the arms are held alongside one another, truly identical positions down each arm are perceived to be different in a direction opposite to that of the previous fusional adjustment. Re-exposing subjects to true spatial relationships (probe opposite button) results in a decay of the aftereffect over trials, although the present ratio of fusion/nonfusion trials $(20 / 10)$ does not permit a change of sufficient magnitude to reach the spatial equivalence shown by control subjects.

Experiments 2 and 3 show that these results are not to be explained by reference to postural or joint adaptation or aftereffects. Thus, the treatment pro- 
duces no change in the perceived orientation of the shoulders or the registered degree of flexion of the used finger.

We have also learned that, when the index finger of the right hand is kept upon the button, up to a 4-sec delay in the onset of probing the left wrist does not cancel the experience of fusion between points on the two arms, which are separated by 4 in. $(10.2 \mathrm{~cm}){ }^{3}$ This suggests that the brain does not require the simultaneous presence of information associated with efference to the active arm and afferent input from the disparate location defined by the probe. We have yet to discover whether muscular activity has any relevance to kinesthetic fusion or whether the presence of spatially discordant tactile information from the two arms is the major variable.

Subsequent to the treatment, it was not uncommon for subjects to report spontaneously that they felt the pointing (active) arm to be shorter than the other. Attempts were often made to rationalize the sensation: thus, "I see what is going on; the switch (button) is gradually moving closer to me and that is why my right arm feels like this." At other times, puzzlement was expressed: "It is funny you know, you're getting hit in the same spot each time yet the finger (right hand) feels to be in different places." There were fewer comments about the probed arm, but one subject reported that the "opposite" probe (which was hitting the palm) was touching her at a point beyond the tips of her fingers.

These experiments make it clear that a kinesthetic fusion phenomenon exists and occurs in response to spatial discordance. It is appropriate to ask what specifies discordance in this situation. It is not enough to argue that it is merely a difference between fingertip and probed spot, for a finger could be (and often is) in any location while another part of the body is being touched. The two events are not normally functionally correlated. In this case, however, they are, and thus the following view is suggested. When two parts of the body are analyzed by the brain as being in the same general region of space, and, for whatever reason, sensory inflow from them is also analyzed to be causally interdependent, then any spatial differences will be processed as discordance. Following the argument in the introduction, the system will thus seek to eliminate the discordance by parametric adjustment of the spatial sense(s) involved. We see this process, therefore, as essentially similar to that involved in prism adaptation, in which perceived spatial discordance results in adaptation of a relevant spatial domain. In the present case, the dis- cordance between right index finger and probed spot that results from tactile stimuli leads to adaptation of a mechanism in the underlying length domain.

Taken together, the preceding data, the comments of the subjects, and the above argument all suggest that, when kinesthetic fusion occurs, registered arm length is being manipulated.

\section{REFERENCES}

Brueld, J. H., \& Albee, G. W. Effect of asymmetrical retinal stimulation on the perception of the median plane. Perceptual and Motor Skills, 1955, 5, 133-139.

Craske, B., \& Crawshaw, M. Spatial discordance is a sufficient condition for oculomotor adaptation to prisms: Eye muscle potentiation need not be a factor. Perception \& Psychophysics, $1978,23,75-79$.

How ARd, I. P., \& Templeton, W. B. Human spatial orientation. New York: Wiley, 1966.

KENNY, F. T. Experimental modification of the knowledge of limb-length: A study of kinesthesis. Unpublished doctoral dissertation, Memorial University of Newfoundland, 1978.

Nachmias, J. Figural after-effects in kinesthetic space. American Journal of Psychology, 1953, 66, 609-612.

PARK, J. N. Displacement of apparent straight ahead as an aftereffect of deviation of the eyes from normal position. Perceptual and Motor Skills, 1969, 28, 591-597.

Radeau, M., \& Bertleson, P. The after-effects of ventriloquism. Quarterly Journal of Experimental Psychology, 1974, 26, 63-71.

Welch, R. B. Perceptual modification: Adapting to altered sensory environments. New York: Academic Press, 1978.

\section{NOTES}

1. It is commonly accepted that the evidence of our eyes is potent (Welch, 1978); in this case, it would seem that the crossmodal information (visual/tactile and visual/kinesthetic for left and right arms, respectively) is more powerful in leading to a veridical perceptual model of the world than is the intramodal discordance between right-fingertip and left-arm probe.

2. Clearly, if fusion is the result of experiencing the trunk to be twisted to the right (which allows the brain to analyze the right finger to be opposite to the probed spot), the subsequent measures of standing normally (not feeling the trunk to be twisted) or judgments of the arms as being straight out will reveal the joint or postural AE.

3. Since we wish to know whether the experience of kinesthetic fusion is due to postural/joint adaptation, it was appropriate to modify the experimental conditions to minimize the possibility of their occurrence. An absence of postural effects in the presence of kinesthetic fusion was the goal of the manipulation, as it could establish kinesthetic fusion as a phenomenon sui generis.

4. This is reasonable, because, in this experiment, subjects who show any change in the perceived distance between finger and probe give decreasing estimates within a block with no reversals; thus, the final estimate is always the lowest (i.e., exhibits the most fusional shift).

5. Informal observation indicates that even 15-sec delay does not preclude the fusion experience.

(Received for publication March 24, 1980; revision accepted March 14, 1981.) 